\title{
DEVELOPMENT OF PIPE CRAWLING ROBOTS WITH VIBRATORY DRIVES AND INVESTIGATION OF THEIR KINEMATIC PARAMETERS
}

\author{
Vygantas Mištinas ${ }^{1}$, Bronislovas Spruogis ${ }^{2}$ \\ Department of Transport Technologic Equipment, Vilnius Gediminas Technical University, \\ Plytines g. 27, LT-2040 Vilnius, Lithuania \\ Received 200205 27; accepted 20020910
}

\begin{abstract}
In the article the classification of pipe crawling robots with vibratory drives is presented, their advantages and imperfections are reviewed. The schemes of the construction of a pipe crawling robot with a vibratory impulse drive and a pipe crawling robot with a vibratory pneumatic drive are discussed. The dynamic models of a robot with a vibratory impulse drive and of a robot with a vibratory pneumatic drive were developed and the equations of their movement were formed and written.
\end{abstract}

Keywords: a pipe crawling robot, a vibratory drive, a vibratory impulse drive, a vibratory pneumatic drive, piping system, dynamic model.

\section{Introduction}

The pipe transport is widely used for the transportation of homogenous substances (such as water, gas, oil and its products, various chemicals and so on). In the Republic of Lithuania the length of pipes for oil transportation is only over $400 \mathrm{~km}$. In a course of time a pipe wears out, i.e. the inner surface of the pipe is covered by corrosion coating, the welding seams of the pipes lose their tightness, defects in the structure of the pipe material appear and a layer of thin coating is formed on the inner surface of the pipe during transportation of various sticky substances, thus reducing the capacity of the pipe (Only due to the transportation of oil and its products this thin coating constitutes to $70 \%$ of the diameter of the pipe).

After an earthquake or bombing, rescuers who climb into the rubble of collapsed buildings searching for survivors may place their own life at risk, as well as the life of unseen survivors hidden deeper beneath the rubble $[1,2]$.

So, special technical means are necessary to control pipes. Lately robots are world-widely used for the control of the inner part of pipes.

\section{The Classification of Pipe Crawling Robots}

Piping systems for the transportation of substances are classified according to the diameters of pipes, length of pipes, transportable substances, radii of pipe bending and so on. Many various robots are developed for the control of inner surface of cylindrical pipes $[3,4]$. They are divided into groups according to the manner of driving of

\footnotetext{
${ }^{1}$ E-mail: mvygis@one.1t

²E-mail: tti@ti.vtu.lt
}

the robot in the pipe:

- self-propelled;

- drawn with a rope or chain;

- moving together with the transported substance;

- of a combined type.

According to their functional purpose robots are classified to:

- diagnostic

- cleaning

- combined (diagnostic and cleaning)

In their turn, self-propelled pipe crawling robots are classified:

1. according to periodicity of movement:

- $\quad$ step movement;

- continuous movement

2. according to the type of the drive:

- electric;

- hydraulic;

- pneumatic;

- magnetic;

- combined

3. according to the interaction with the surface of support:

- $\quad$ interacting with a plane;

- interacting with a deformation-proof pipe;

- interacting with deformable media.

In their turn, step movement pipe crawling robots are classified according to the type of control to:

- programmed control;

- biotechnical;

- intellectual.

According to the method of cleaning of pipes the pipe crawling robots are classified to:

- mechanical; 
- chemical;

- combined (mechanical and chemical).

A self-propelled pipe crawling robot consists of an open kinematic circuit which links are connected to each other with kinematic couples. Crawling contact surface is formed by rollers, cater-pillar tracks and so on of the corresponding shapes. All self-propelled continuous movement pipe crawling robots may be divided into two groups according to their design.

The first group [5] consists of a carrying frame with the robot moving a drive mounted on it, the end link of the drive being a roller connected with another roller or caterpillar track in a kinematic way. On the top of the robot a support link is mounted which is connected in a kinematic way with the carrying frame via a cylindrical reductor and a screw-nut drive. The support link is used to ensure the stability of the robot. The support link with a support roller mounted on its end may hinge by an angle to $90 \mathrm{r}$ and move around the axle of fitting. This ensures the stability of movement of the robot on changes of the diameter of the pipe.

The principle of movement of mechanisms of this group is the following: the pipe crawling robot moving drive via the kinematic link transfers a rotational movement to the driving roller which rotates the roller or caterpillar contacting with the inner surface of the pipe. The position of the support link alters dependently on the diameter of the pipe and on changes of the latter. The changes of the position take place as well.

The advantages of this group of design of pipe crawling robots are the following:

1. Self-propelling.

2. Good control of the speed of movement and the force of cohesion with the contact surface.

3. Good capacity of piping where diameters of pipes are different.

4. Sufficient capacity in bent fragments of piping.

The imperfections of this group of design of pipe crawling robots are the following:

1. They can be used only after the termination of supply of the transported substance.

2. Limited possibilities of power supply.

3. Rotational movement on the contact with the pipe.

The second group of continuous movement pipe crawling robots includes "rotating" mechanisms [6]. Such mechanism consists of a stator with a rotor put on it. A brake is fitted between the stator and rotor. Cuts may be provided in the rotor for the injection of the substance for cleaning the inner surface of the pipe. The substance may be held in the rotor mechanism itself or supplied by hoses.

The principle of operation of the pipe crawling robot is the following: when the rotor with spiral-shaped notches, which enter into contact with the pipe, is rotating on the stator, the robot is moving forward or backward dependently on the direction of rotation of the rotor. If several such rotors are connected with each other, the mobility of robots of this group increases considerably in bent frag- ments of the piping.

The advantages of this group of designs of pipe crawling robots are the following:

1. Self-propelling.

2. Good control of the speed of movement and the force of cohesion with the contact surface.

The imperfections of this group of construction of pipe crawling robots are the following:

1. They can be used only after the termination of supply of the transported substance.

2. Limited possibilities of power supply.

3. Limited capacity in bent fragments of piping.

4. Rotational movement at the contact with the pipe.

5. Limited capacity of piping with different diameters of pipes.

Self-propelled step movement pipe crawling robots also consist of an open kinematic circuit which links are connected to each other with kinematic couples and in the drive of power supply a supplemental movement transforming equipment is provided. According to the peculiarities of design all the self-propelled step movement pipe crawling robots may be divided into three groups.

Pipe crawling robots of the first group [7] consist of links connected with cylindrical inter-link hinge joints. Each hinge joint is connected to electric engine via the worm reductor and one-side clutch. An angle meter for the measurement of the angle between links is mounted into each hinge joint. One of the middle links consists of two parts connected with a hinge joint. These parts are also connected with a cylindrical hinge joint which is connected in a kinematic way with self-stopping worm reductor and electric engine. A rotation angle measuring device is mounted into the inter-link hinge joint. The axle of this joint is perpendicular to the axles of the inter-link cylindrical hinge joints and parallel to the longitudinal axle of the pipe.

The principle of operation of the construction of this group is the following: the movement of links around the cylindrical hinge joint, perpendicular to the longitudinal axle of the piping, enables the drive of each link to change positions of links of the robot in the longitudinal plane of the pipe in order to form bent elbows. In such case the support pressure on the contact ensures the sufficient friction force (on rest) to achieve immobility of the bent elbow and free movement of other links. Because the length of the bent elbow is less than the one of the straightened links, forming the elbow, the bending and straightening of the elbow make the pipe crawling robot move. Such pipe crawling robot may move according to the principle of "running wave", when the functions of formation of bent elbow and the change of its length are bound and the bent elbows are formed in succession along the total length of the mechanism. Such mechanism may also move with the fixed front and end bent elbows and change the length of the middle links on changes of the fixation.

The advantages of this group of design of pipe crawling robots are the following:

1. Self-propelling.

2. No motion at the contact with the pipe. 
1. Good control of the speed of movement and the force of cohesion with the contact surface.

2. Good capacity in bent fragments of piping.

3. Good capacity of piping, where diameters of pipes are different.

The imperfections of this group of construction of pipe crawling robots are the following:

1. They can be used only after the termination of supply of the transported substance.

2. Limited possibilities of power supply.

3. Limited movement when the layer of coating on the inner surface of the pipe is too thick.

The second group of step movement pipe crawling robots includes robots with a pneumatic drive [8] (See Fig 1).

The advantages of this group of design of pipe crawling robots are the following:

1. Self-propelling.

2. No motion at the contact with the pipe.

3. Good control of the speed of movement and the force of cohesion with the contact surface.

The imperfections of this group of construction of pipe crawling robots are the following:

1. They can be used only after the termination of supply of the transported substance.

2. Limited possibilities of power supply.

3. Limited movement when the layer of coating on the inner surface of the pipe is too thick.

1. Limited capacity in bent fragments of piping.

2. Limited capacity of piping with different diameters of pipes.

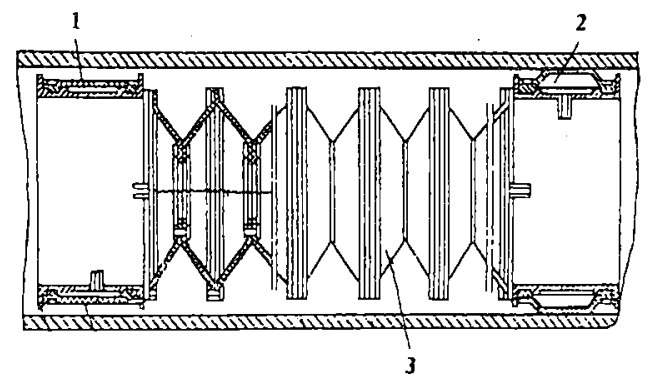

Fig 1. The scheme of transporting blocks of a crawling robot with deformable pressing elements:

1,2 - the transporting blocks, 3 - the deformable pressing element

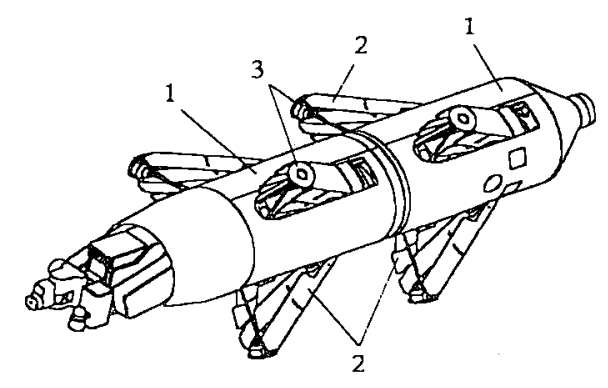

Fig 2. The scheme of transporting blocks of crawling robots with unbendable feet

1-the transporting blocks, 2 - the unbendable feet, 3 - special surfaces
The third group of step movement pipe crawling robots includes robots with an electric drive [9] (See Fig 2). Their constituent parts are the following: a central body with electric engines mounted on it which are comected with the movement supports. The number of the supports is no less than six, i.e. three supports in one plane and three supports in the other plane. In this way the stability of the movement of the robot is ensured. The number of the supports may be increased in order to improve the permeability of the robot when the layer of coating on the inner part of the pipe is too thick.

The principle of operation is the following: the electric engine mounted on the central body activates a step movement of the supports and thus enables the pipe crawling robot to move forward or backward.

The advantages of this group of construction of pipe crawling robots are the following:

1. Self-propelling.

2. No motion at the contact with the pipe.

3. Good control of the speed of movement and the force of cohesion with the contact surface.

The imperfections of this group of construction of pipe crawling robots are the following:

1. They can be used only after the termination of supply of the transported substance.

2. Limited possibilities of power supply.

3. Limited movement when the layer of coating on the inner surface of the pipe is too thick.

4. Limited capacity in bent fragments of piping.

5. Limited capacity of piping with different diameters of pipes.

\section{New Schemes of Design of Pipe Crawling Robots}

After the analysis of the peculiarities of the design of the pipe crawling robots $[10-11]$ it was decided to develop new schemes of design of the robots, preserving good permeability of the robots in bent fragments of piping and when the diameters of pipes are different as well as other features of the existing pipe crawling robots. The new schemes are presented in Fig 3.

a)
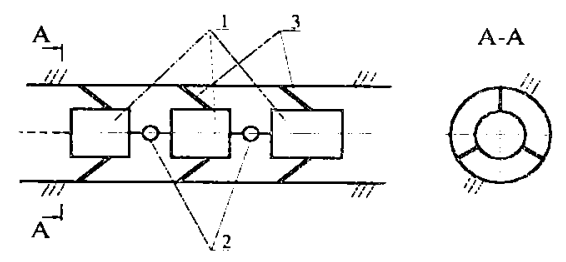

b)
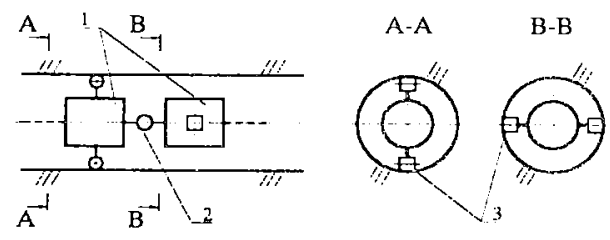

Fig 3. The schemes of crawling robots for curved pipes: a) the principal scheme of the drive, b) the fragments of the drive's element; 1 - the element of the vibratory drive, 2 - the elastic hinge joints, 3 - the hinge joint elastic contact supports 
A robot with vibratory drives in its transporting block is classified as a robot of a newer type (See Fig 3). A drive of the transporting block of such robot consists of excitable masses, elastic elements and electrostatic or pneumatic exciting elements which excite vibrations of the transporting blocks (Fig 3). So, the transporting block may be called the exciting and transporting block. The electrostatic exciting elements via the elastic elements vibrate the excitable masses which develop a friction force at the contact with the support and push the transporting block. Because of this the transporting block starts to move. Robots with transporting blocks of this type may be of smaller dimensions, they are more mobile, so their field of application is more various and wider.

\subsection{Pipe Crawling Robots wiih a Vibratory Pneu- matic Drive}

A pipe crawling robot with a pneumatic drive consists of $n$ sections with autonomous feeding; the sections are interconnected with elastic hinge joints, and in addition, the body of each section is fixed to one-stage elastic hinge dampers with $120^{\circ}$ angle between them that bend at a certain angle with the direction opposite to the one of the movement; an elastic element is fixed between the said dampers and the side surface of the body.

The scheme of a pipe crawling robot with a pneumatic drive is presented in Fig 4.

A pipe crawling robot with a pneumatic drive consists of $n$ sections interconnected with elastic hinge joints. Each section consists of the body I with three one-stage elastic hinge dampers 2 with $120^{\circ}$ angle between them fixed to it that are bent at the angle a against the direction opposite to the one of the movement. No less than two rows of such dampers must be provided.

In order to ensure the unidirectional movement of the robot between the body 1 and the inner surface of the piping system as well as the contact surface of the dampers 2, a roll is mounted on the end of the damper. In the end part of the body the elastic element 4 is fixed and the piston 5 is fixed to its other end. The branch pipes 6 and 8

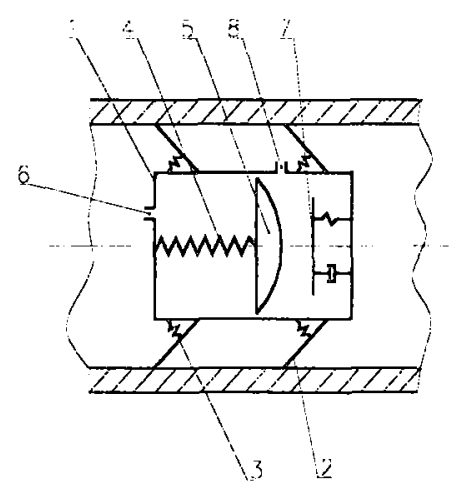

Fig 4. The scheme of the design of the original step movement robot with a vibratory pneumatic drive:

1 - the movement support, 2, 7 - the damper, 3, 4-the elastic element, 5 - the piston, 6 - the feeding branch pipe, 8 the released branch pipe for air supply and release are provided in the end and side parts of the body. Various diagnostic and repairing equipment may be fixed to the robot.

The compressed air is supplied from the pneumatic system receiver via the feeding branch pipe 6 that causes vibrations of the piston 5 through the elastic element 4 . Because of this, the piston 5 contacts with the elastic element 3 fixed in the damper 2 and the element enables the dampers to be elastic and to change the trajectory of the movement in the piping, if the inner diameter of the piping as well as the thickness of the coating of the inner surface of the piping vary. In order to ensure a reliable movement of the robot along the inner surface of the piping system, it is necessary to ensure the rigidity of the one-stage elastic hinge dampers 2 higher than the rigidity of the elastic elements 3 . In order to reduce the friction on the contact surface between the inner surface of the piping system and the contact surface of the dampers 2 , on the end of the damper a roll may be mounted. In the end part of the body the elastic element 4 is fixed which other end is connected to piston 5. The branch pipes 6 and 8 are provided in the end and side parts of the body for air supply and release. Various diagnostic and repairing equipment may be fixed to the robot.

The compressed air is supplied from the pneumatic system receiver via the feeding branch pipe 6 that causes vibrations of piston 5 through the elastic element 4 . Because of this piston 5 in the contact with damper 7 overcomes the force of friction of the robot against the wall of the pipe and pushes a separate section of the robot. At that moment the compressed air is released from branch pipe 8 . This causes starting of the movement of all sections of the robot and the whole piping system with the pneumatic drive starts moving.

At the examination of the pipe crawling robot with a vibratory impulse drive the following dynamic model of this scheme was developed (See Fig 5) [10-12]:

$$
x_{1}^{\prime}>0
$$

when $x_{2}<x_{3}$;

$$
\begin{aligned}
& m_{1} x_{1}^{\prime \prime}+P_{1}+F_{12}+F_{f}=F_{x 1} ; \\
& m_{2} x_{2}^{\prime \prime}+P_{2}+F_{21}=0 ; \\
& F_{3}=0 ; \\
& \Delta Q=0 ; \\
& \text { when } x_{2}=x_{3} ; \\
& m_{1} x_{1}^{\prime \prime}+P_{1}+F_{12}+F_{f}-F_{3}=F_{x 1} ; \\
& m_{2} x_{2}^{\prime \prime}+P_{2}+F_{21}+F_{3}=0 ; \\
& \Delta Q=0 ; \\
& x_{1}^{\prime}=0 ;
\end{aligned}
$$


when $x_{2}<x_{3}$;

$$
\begin{aligned}
& m_{2} x_{2}^{\prime \prime}+P_{2}+F_{21}=0 ; \\
& F_{3}=0 ; \\
& \Delta Q=0 ;
\end{aligned}
$$

when $x_{2}=x_{3}$;

$$
\begin{aligned}
& m_{2} x_{2}^{\prime \prime}+P_{2}+F_{21}+F_{3}=0 \\
& \Delta Q=0
\end{aligned}
$$

where $m_{1}, m_{2},-$ masses; $x_{1}, x_{2}, x_{3}$-shifts of the robot and its masses, $H$-coefficient of resistance of a linear shift.

$$
\begin{aligned}
& F_{12}=-F_{21}=H_{12}\left(x_{1}^{\prime}-x_{2}^{\prime}\right)+c_{12}\left(x_{1}-x_{2}\right) ; \\
& F_{3}=H\left(x_{3}^{\prime}-x_{1}^{\prime}\right)+c\left(x_{3}-x_{1}-x_{s}\right) .
\end{aligned}
$$

After the solution of these equations and analysis of the solutions as well as the optimization of the parameters of the dynamic model it will be possible to present the optimum design of the activator of vibrations.

\section{Drive}

\subsection{Pipe Crawling Robots with a Vibratory Impulse}

The robot with a vibratory impulse drive (Fig 6) consists of the body 1 with three supports 2 fitted on it, forming an angle of 120

In order to ensure the movement of the robot in one direction the elastic element 3 is inserted between the body 1 and the support 2 . In the end part of the body an alternating electromagnet 4 is mounted with the elastic element 5 and damper 6 fitted to it. A moving mass (permanent magnet) is connected to the elastic element 5 and damper 6.

Various diagnostic and cleaning equipment may be connected to the robot.

At the examination of the pipe crawling robot the following dynamic model of this scheme was developed (See Fig 7):

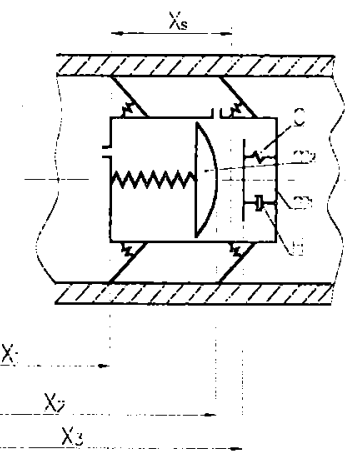

Fig 5. The dynamic model of the original pipe crawling robot with vibratory pneumatic drive:

$m_{2}$ - the moving mass, $m_{1}$ - the mass of the body, $x_{1}$ - the shift of the robot, $x_{2}$ - the shift of the moving mass, $x_{3}-$ the shift of the moving mass till the damper; $\mathrm{C}$ - the coefficient of stiffness, $\mathrm{H}$ - the coefficient of resistance of linear shift

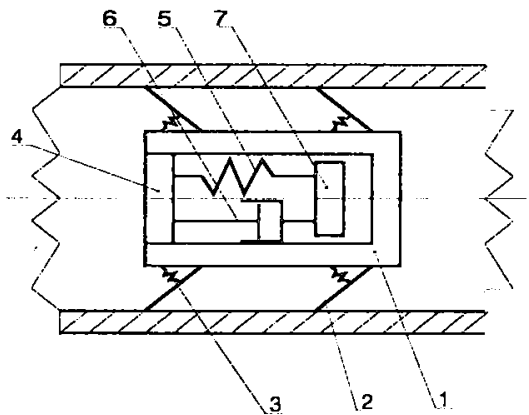

Fig 6. The scherne of the design of the original step movement robot with a vibratory impulse drive:

1 - the body, 2 - the movement support, 3 - the elastic elements, 4 - the alternating-current electromagnet, 5 - the elastic element, 6 - the damper, 7 - the moving mass (permanent magnet)

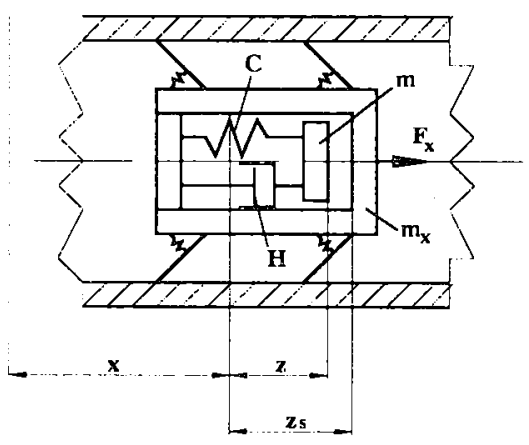

Fig 7. The dynamic model of the original pipe crawling robot: $m$ - the moving mass, $m_{x}$ - the mass of the body, $x$ - the shift

of the robot, $z$ - the shift of the moving mass,

$\mathrm{C}$ - the coefficient of stiffness, $\mathrm{H}$ - the coefficient of resistance of linear shift

The mathematical expression of the dynamic model of the system shall be the following system of equation [10-12]:

When $\mathrm{z}<\mathrm{z}_{\mathrm{s}}$;

$$
\left\{\begin{array}{l}
x^{\prime \prime}+z^{\prime \prime}+h z^{\prime}+h_{2} z=f_{0} \sin w t \\
\mu x^{\prime \prime}+f_{f}-h z^{\prime}-h_{2} z=f_{f}
\end{array}\right.
$$

when $\mathrm{z}=\mathrm{z}_{\mathbf{s}}$;

$$
\begin{aligned}
& \left\{\begin{array}{l}
z^{\prime+}=R z^{-} \\
x^{\prime+}=x^{\prime-}+\frac{H R}{1+\mu} z^{\prime-}
\end{array}\right. \\
& 0<R<1 ; \mu=\frac{m_{X}}{m} ; \quad h=\frac{H}{m} ; h_{2}=\frac{C}{m} ; \\
& f_{0}=\frac{F_{0}}{m} ; f_{f}=\frac{F_{f}}{m} ;
\end{aligned}
$$

where $m_{x^{\prime}} m$-masses; $x, z$-shifts of the robot and its masses; $C$ - coefficient of stiffness; $H$ - coefficient of resistance of a linear shift; $z^{\prime+}, x^{+}-$the speed and the shift after a impulse, respectively; $z^{\prime-}, x^{-}$- the speed and the shift before a impulse, respectively; $f_{0^{\prime}} f_{f}$ - the coefficients 
of dry and liquid friction

After the solution of these system equations including friction force of the robot an effect upon the wall of the pipe obtained these dynamical parameters of the system (See Fig 8-11):

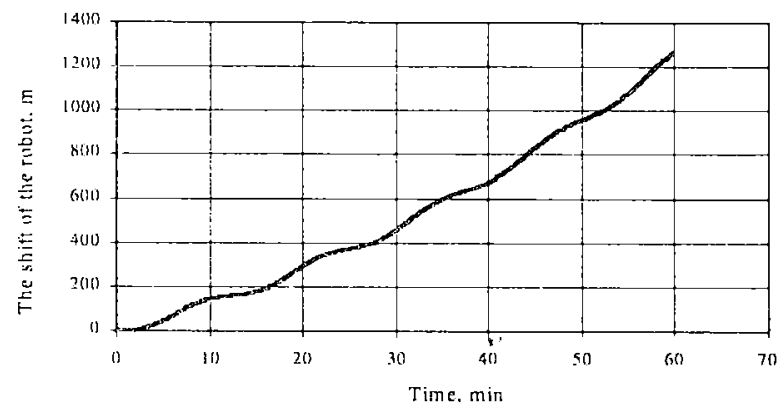

Fig 8. The shift of the robot during the time

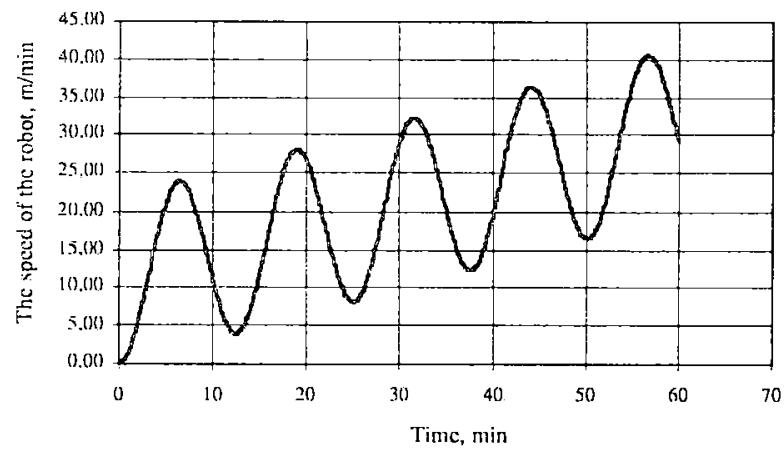

Fig 9. The speed of the robot during the time

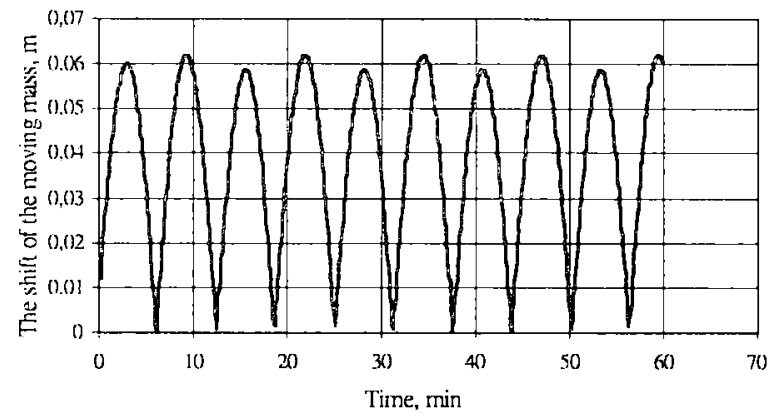

Fig 10. The shift of the moving mass during the time

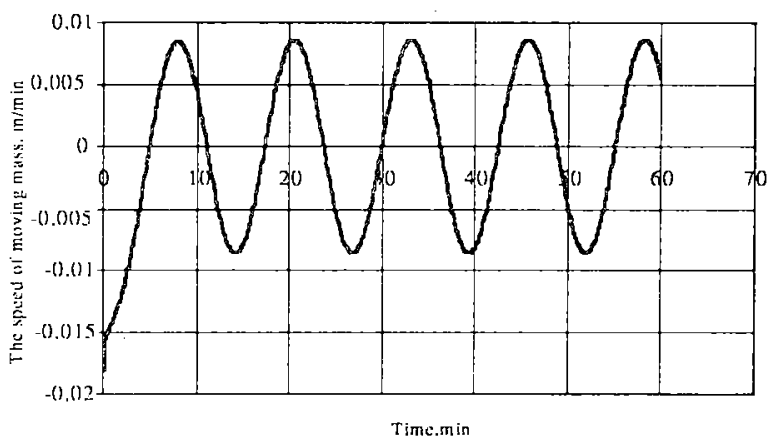

Fig 11. The speed of the moving mass during the time

\section{Conclusion}

1. The review of designs of pipe crawling robots moving by curved trajectories and their several new schemes are presented.

2. The model of the robot with a vibratory impulse drive with its dynamic characteristics was developed.

3. The model of the robot with a vibratory pneumatic drive with its dynamic characteristics was developed.

\section{References}

I. Wapenhans H., Steinle J., Pfeiffer F. Optimal Trajectory Planning with Application to Industrial Robots. Journal of Advanced Manufacturing Technologies, London: Springer Verlag, 1994, 9, S 49 - 55.

2. Glocker Ch., Pfeiffer F. Multiple Impacts with Friction in Rigid Multibody Systems. In: Proc. of the 5th Conf. on Nonlinear Vibrations, Stability, and Dynamics of Structures and Mechanics, Blacksburg, VA, USA, Vol 5, 1994, p 85 89.

3. Pfeiffer F., Rosmann T. Control of a tube crawler. Movic '98. Zurich. Switzerland. August 25 - 28, 1998, Vol 3, p 889 - 894.

4. Pfeiffer F., Rosmann J. Control and design of a Pipe Crawling Robot. In: Proc. of the $13^{\text {th }}$ World Congress, Int. Federation of Automatic Control, San Francisko, USA, 1996, p. 159-165.

5. Pipe cleaning equipment. The USSR Author's Certificate No 1739729. Published in Bull. (Бюллетень изобретений), No 19, Moscow, 1995, p 1 - 6 (in Russian).

6. Pipe cleaning equipment. The Patent of Germany No 19524459A1. Inventions of states of the World (Изобретения стран мира), Issue 14, No 2, Moscow, 1998, p 14 - 15 (in Russian).

7. The equipment for welding inner surface of pipes. The USSR Author's Certificate No 1026996. Published in Bull. (Бюплетень изобретений), No 25, Moscow, 1983, p 1 - 3 (in Russian).

8. The equipment for a defectoscopy of the inner surface of piping systems. The USSR Author's Certificate No 849058. Published in Bull. (Бюллетень изобретений), No 27, Moscow, 1981, p 1 - 5 (in Russian).

9. Pipe cleaning equipment. The Patent of USA No 5636403A Inventions of states of the World. (Изобретения стран мира), Issue 14, No 9, Moscow, 1998, p 15 - 16 (in Russian).

10. Matuliauskas A., Mištinas M., Spruogis B., Ragulskis K. Pipe crawling robots with vibratory drives. In: Proceedings of the $2^{\text {nd }}$ International Anniversary Conference VIBROENGINEERING-2000, 21 September 2000. Journal of Vibroengineering, No 4 (5), Vilnius, Lithuania, 2000, p 9-14.

11. Kulvietis G., Matuliauskas A., Mištinas M., Spruogis B., Ragulskis K. Dynamic Parameter Investigation of Pipe robots. In: Proceedings of the $2^{\text {nd }}$ International Anniversary Conference VIBROENGINEERING-2001, 21 September 2001. Journal of Vibroengineering, No 2 (7), Vilnius, Lithuania, 2001, p 55-58.

12. Žiliukas P., Barauskas R. Mechanical vibration (Mechaniniai virpesiai). Kaunas: Technologija, 1997.310 p. (in Lithuanian). 